\title{
HSCCC Separations of Rutin Esters Obtained by Enzymatic Reaction Catalyzed by Lipase
}

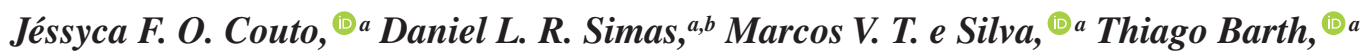 \\ Shaft C. Pinto, ${ }^{\oplus a}$ Luzineide W. Tinoco, ${ }^{\circledR c}$ Denise M. G. Freire, ${ }^{d}$ Michelle F. Muzitano ${ }^{\circledR a}$ \\ and Ivana C. R. Leal ${ }^{\circledR} * a, b$ \\ ${ }^{a}$ Laboratório de Produtos Bioativos (LPBIO), Faculdade de Farmácia, \\ Universidade Federal do Rio de Janeiro, 27933-378 Macaé-RJ, Brazil \\ ${ }^{b}$ Laboratório de Produtos Naturais e Ensaios Biológicos (LaProNEB), \\ Departamento de Produtos Naturais e Alimentos, Faculdade de Farmácia, Centro de Ciências da Saúde, \\ Universidade Federal do Rio de Janeiro, Ilha do Fundão, 21941-902 Rio de Janeiro-RJ, Brazil \\ 'Instituto de Pesquisas de Produtos Naturais Walter Mors, Centro de Ciências da Saúde, \\ Universidade Federal do Rio de Janeiro, Ilha do Fundão, 21941-590 Rio de Janeiro-RJ, Brazil \\ ${ }^{d}$ Laboratório de Biotecnologia Microbiana, Instituto de Química, Centro de Tecnologia, \\ Universidade Federal do Rio de Janeiro, Ilha do Fundão, 21941-590 Rio de Janeiro-RJ, Brazil
}

\begin{abstract}
The flavonoid rutin presents several pharmacological effects, despite this, its application in the pharmaceutical industry can be significantly limited by its low bioavailability. The development of lipophilic derivatives by esterification of hydroxyl groups with fatty acid chains may be an effective strategy to change their physicochemical properties. This work aims to use high-speed countercurrent chromatography (HSCCC) to isolate rutin esters produced by esterification reactions catalyzed by the immobilized lipase from Candida antarctica (Novozyme $435^{\circledR}$ ). The lipasecatalyzed synthesis of rutin esters (R3-R18:2) in 2-methyl-2-butanol exhibited conversions that ranged from 16 to $40 \%$ and highest conversion for short chain fatty acids (R4-R12). After initial partitioning tests of the reaction mixture in different solvent proportions followed by thin layer chromatography (TLC) analysis, the biphasic solvent systems consisting in HEMWat (hexane/ ethyl acetate/methanol/water) in different proportions were chosen to separate rutin esters. These esters were separated for the first time to the reaction mixture via HSCCC. This technique proved to be more advantageous than the traditionally one since it allowed quick isolation and high purity $(\geq 90 \%)$ of the products. It also permitted a substrate recovery for reuse in other enzymatic reactions. Furthermore, the results add valuable information, specially concerning the structures elucidation of different rutin esters not previously described.
\end{abstract}

Keywords: countercurrent chromatography, esterification reaction, fatty acids, Novozyme, rutin esters

\section{Introduction}

Flavonoids are an important natural product group widely distributed in the plant kingdom. ${ }^{1}$ Different subgroups of flavonoids can be formed depending on the connection of the aromatic ring to the heterocyclic ring and, on the degree of unsaturation and oxidation of the $\mathrm{C}$ ring. The rutin (3', 4',5,7-tetrahydroxyflavone-3 $\beta$-D-rutinoside) is a quercetin $O$-glycoside flavonoid that presents in position 3 of the $\mathrm{C}$ ring an unit of rutinose (glucose and rhamnose; $\operatorname{Ram} \alpha(1 \rightarrow 6) \mathrm{Glc}){ }^{2}$

*e-mail: ivanafarma@yahoo.com.br, ivana@pharma.ufrj.br
Rutin has already been associated with various pharmacological potentials including antioxidant, antimicrobial, anti-inflammatory, anticancer, antidiabetic, antiallergic and neuroprotective activities. ${ }^{1,3}$ Despite this, the efficacy of rutin can be significantly limited by its low bioavailability. ${ }^{1}$

The development of lipophilic derivatives of rutin through the esterification of hydroxyl groups with fatty acid chains can be an effective strategy to modify their physicochemical properties and, likely, to favor their bioavailability. Furthermore, this structural modification can be a strategy to improve the pharmacological activity of the generated ester compared to the original compound. ${ }^{4,5}$ 
The esterification reaction can be carried out by enzymatic pathways, processes known as biocatalysis. The biocatalytic method presents several advantages compared to the classical chemical synthesis such as obtaining the desired product under milder reaction conditions of $\mathrm{pH}$ and temperature, with higher purity. The use of immobilized enzymes can also allow the reuse of the biocatalyst by many cycles, depending on the stability, considerably reducing process cost. ${ }^{6,7}$

Among the enzymes able to conduct esterification reactions are the lipases, an important group classified as triacylglycerol acyl hydrolase (Enzyme Commission, EC 3.1.1.3) that catalyzes the hydrolysis of ester bonds. However, in low-water environments, lipases are able to reverse the reaction, resulting in esterification steps. ${ }^{8}$ The Candida antarctica lipase B (CALB) is an enzyme already well-studied as a biocatalyst for esterification of a wide range of substrates, such as glycosylated flavonoids, allowing a process with good catalytic activity in a nonaqueous media, resistance to thermal inactivation and a high degree of enantio- and regioselectivity. ${ }^{4}$

Regioselectivity studies of CALB demonstrated that the aglycon portion of flavonoids is stabilized at the entrance of the enzyme cavity by hydrogen bonds and hydrophobic interactions involving phenolic groups, locating their glycoside portions near to the catalytic residues of the enzyme active site. ${ }^{9}$ Rutin reactions catalyzed by CALB have been reported and the esters generated are those at 4"'-OH position of rhamnose, ${ }^{10-17}$ or 3"- $\mathrm{OH}$ position of glucose, ${ }^{18,19}$ or in both, ${ }^{20}$ or still in both 4 "'-OH and 4 "' $-\mathrm{OH}$ positions. ${ }^{21}$

To date, the most usual method to isolate esters of flavonoids of the reaction mixture are column chromatography using silica gel, ${ }^{14-17,19-27}$ preparative high-performance liquid chromatography (HPLC), ${ }^{12,13,28}$ traditional liquid-liquid extractions, ${ }^{10,11,29,30}$ or preparative thin layer chromatography (TLC). ${ }^{22,31}$ However, these techniques have presented some disadvantages such as high solvent consumption, irreversible adsorption of the sample and low sample loading. Concerning the traditional liquidliquid extraction there is also a difficulty for establishing a solvent system that allows fast separation of the products with different lipophilic chains.

Countercurrent chromatography (CCC) is a liquidliquid separation technique, in which the liquid stationary phase is maintained in the column by centrifuge force while the immiscible mobile phase flows through it. ${ }^{32}$ This process, widely used for the isolation of flavonoids, has shown advantages such as to admit a high sample loading and a rapid isolation of high purity products. There is also no irreversible adsorption on solid support, enabling full recovery of the sample, proving to be an economical, versatile and reproducible technique. ${ }^{33}$

Despite the growing number of publications using the CCC approach in order to isolate different types of flavonoids whether in glycosylated, methylated, prenylated, galloyled, malonated, or acetylated forms, ${ }^{33}$ it is the first time that this technique is being used to separate rutin ester derivatives constituted by lipophilic chains of different sizes produced by biocatalytic pathway. Therefore, this work aims to develop and apply a method using high-speed countercurrent chromatography (HSCCC) to isolate rutin esters produced by reactions catalyzed by immobilized lipase from Candida antarctica (Novozyme $435^{\circledR}$ ).

\section{Experimental}

\section{General experimental procedures}

Nuclear magnetic resonance (NMR) spectra of the purified product were recorded on a Varian VNMRSYS $500 \mathrm{MHz}$ spectrometer (Varian Inc., Palo Alto, CA, USA) working at $499.78\left({ }^{1} \mathrm{H}\right)$ and at $125.68 \mathrm{MHz}\left({ }^{13} \mathrm{C}\right)$. The pulse sequences used are all standard in the VNMRJ software, and the experiments were conducted at $25{ }^{\circ} \mathrm{C}$ using tetramethylsilane (TMS) as internal standard. The samples were dissolved in dimethyl sulfoxide (DMSO- $d_{6}$ ).

The HSCCC separations were conducted on AECS Quattro QuikPrep (Gloucestershire, UK) apparatus equipped with two bobbins containing two polytetrafluoroethylene multi-layer coil each $(4 \times 112 \mathrm{~mL}, 2.1 \mathrm{~mm}$ internal diameter (id)) and $10 \mathrm{~mL}$ sample loop. The rotation speed is adjustable from 0 to $1000 \mathrm{rpm}$. All separations were performed at $860 \mathrm{rpm}$ and $112 \mathrm{~mL}$ coil at $30^{\circ} \mathrm{C}$. A Pump ECP 2010 model (ECOM ${ }^{\circledR}$, Prague, Czech Republic) was used to pump the solvents and the fractions were collected in FC203B model (Gilson ${ }^{\circledR}$, Wisconsin, USA).

High-performance liquid chromatography coupled to diode array detector (HPLC-DAD)

The extent of the reaction was characterized by highperformance liquid chromatography Shimadzu LC-20AT equipped with a DAD detector SPD M20A (Shimadzu ${ }^{\circledR}$, Kyoto, Japan), Kinetex Biphenyl ${ }^{\circledR}$ column $(4.6 \times 100$ mm, $2.6 \mu \mathrm{m})$. The flow rate was set at $0.6 \mathrm{~mL} \mathrm{~min}^{-1}$ and there were injected $2 \mu \mathrm{L}$ of each sample. The UV/DAD detection was monitored at 190-800 $\mathrm{nm}$. The components were separated using a gradient elution protocol consisted of formic acid solution in ultrapure water $(0.1 \%)(\mathrm{A})$ and methanol (B) such as the following: $0 \mathrm{~min}(40 \% \mathrm{~B}) ; 10 \mathrm{~min}$ 
(40\% B); 12 min (100\% B); 30 min (100\% B); $31 \mathrm{~min}$ (40\% B); $40 \mathrm{~min}(40 \% \mathrm{~B})$. The reaction samples were prepared through the dilution of $50 \mu \mathrm{L}$ aliquot reactions in $950 \mu \mathrm{L}$ methanol. The conversion (\%) was calculated following HPLC-DAD analysis as the area of the ester product divided by the total area, multiplied by 100 . All analyzes were carried out in duplicate.

Ultra-high performance liquid chromatography coupled to diode array and mass spectrometry detectors (UHPLC-DADMS/MS)

The reaction samples and isolated esters were analyzed in a Thermo Scientific LCQ FLEET UHPLC system (Thermo Fisher Scientific, Waltham, USA) equipped with a DAD detector coupled to a mass spectrometer with an electrospray ionization (ESI) source (LCQ, Thermo Fisher Scientific, Waltham, USA). The column used was a Kinetex Biphenyl ${ }^{\circledR}$ column $(4.6 \times 100 \mathrm{~mm}, 2.6 \mu \mathrm{m})$ at $40{ }^{\circ} \mathrm{C}$. The components were separated using the same elution protocol as described before. The flow rate was set at $0.6 \mathrm{~mL} \mathrm{~min}^{-1}$ and, for the analyses, there were injected $2 \mu \mathrm{L}$ of the reaction mixtures and $5 \mu \mathrm{L}$ of the isolated esters. For qualitative analysis of the reaction mixtures, the samples were prepared through the dilution of $50 \mu \mathrm{L}$ aliquots of each reaction in $950 \mu \mathrm{L}$ methanol. The isolated esters were dissolved in methanol $\left(1 \mathrm{mg} \mathrm{mL}^{-1}\right)$ for purity analysis. The experiments were monitored at 210, 254 and $355 \mathrm{~nm}$; and by MS. MS measurements were carried out with helium (99.999\% purity) as the collision gas in the ion trap and nitrogen as the sheath, sweep and auxiliary gas in the source. MS parameters were tuned as electrospray negative ionization mode; the capillary temperature set at $275^{\circ} \mathrm{C}$, source voltage at $5.50 \mathrm{kV}$ and mass range from 500 to 1200 Daltons (reactional mixtures) and from 70 to 1200 Daltons (isolated esters).

\section{Chemicals}

Organic solvents for TLC and CCC analysis were of analytical grade from Tedia ${ }^{\circledR}$ (Rio de Janeiro, Brazil). Organic solvents used in HPLC-DAD (high-performance liquid chromatography coupled to diode array detector) and UHPLC-DAD-MS/MS (ultra-performance liquid chromatography coupled to diode array and mass spectrometry detectors) analysis were of HPLC grade from Tedia ${ }^{\circledR}$ (Rio de Janeiro, Brazil) and ultrapure water provided by a Milli-Q purification system ElgaPurelab ${ }^{\circledR}$ (Wycombe, UK). The organic solvent used for NMR analysis was DMSO- $d_{6}$ (deuterated dimethyl sulfoxide) by $\mathrm{CIL}^{\circledR}$ (Andover, USA). The rutin standard and molecular sieves $4 \AA$ (10-20 mesh) were purchased from Sigma-Aldrich $^{\circledast}$ (St. Louis, USA). 2-Methylbutan-2-ol and fatty acids (propionic, butyric, caproic, caprylic, lauric, myristic, palmitic, stearic, oleic, linoleic acids) were purchased from LobaChemie ${ }^{\circledR}$ (Mumbai, India). The immobilized lipase B from Candida antarctica (Novozyme $435^{\circledR}$ ) used as the biocatalyst was provided by Sigma-Aldrich ${ }^{\circledR}$ (St. Louis, USA). The Natural Product (NP) Reagent A (2-aminoethyl diphenylborinate) was obtained from TCI America ${ }^{\circledR}$ (Tokyo, Japan) and the polyethylene glycol (PEG) was purchased from Tedia ${ }^{\circledR}$ (Rio de Janeiro, Brazil).

\section{Enzymatic synthesis of rutin esters}

Rutin $\left(13.65 \mathrm{mmol} \mathrm{L}^{-1} ; 25 \mathrm{mg}\right)$ and fatty acids $\left(68.25 \mathrm{mmol} \mathrm{L}^{-1}\right)$ were dissolved in the molar ratio 1:5 (rutin:fatty acid) in $3 \mathrm{~mL}$ of 2-methylbutan-2-ol in the presence of $4 \AA$ A molecular sieves $\left(100 \mathrm{~g} \mathrm{~L}^{-1}\right)$. The substrates were previously dried in a desiccator for 7 days while the solvent was dried in the presence of $4 \AA$ molecular sieves for 5 days. After 5 min of sample condition under stirring at $55^{\circ} \mathrm{C}$ and $200 \mathrm{rpm}, 15 \%(\mathrm{~m} / \mathrm{m}$ of total reactants) of immobilized lipase from Candida antarctica (Novozyme $435^{\circledR}$ ) was added to the mixture. The reactions were conducted on an orbital Shaker Solab ${ }^{\circledR}$ SL 223 (São Paulo, Brazil), at $55^{\circ} \mathrm{C}$, by stirring at $200 \mathrm{rpm}$. The aliquots $(300 \mu \mathrm{L})$ were withdrawn at $24,48,72,96$ and $120 \mathrm{~h}$, and initially analyzed by TLC (butanol (4):water (0.25):ethanol (0.25):acetic acid (0.5)) in order to verify qualitatively the product conversion over time, and then by HPLC to quantify the products concerning their relative percentages of conversion based on the peak area on the chromatogram. The final aliquots at $120 \mathrm{~h}$ were also qualitatively analyzed by UHPLC-DAD-MS/MS in order to confirm the production of rutin esters by MS/MS.

All reactions were carried out in duplicate. Blank experiments without the enzyme were also conducted in parallel. Afterwards, the reactions were carried out in a large scale to follow the purification step. The reactions were scaled up in 12-fold by adopting the same proportions of dried rutin $(13.65 \mathrm{mM})$ and fatty acids $(68.25 \mathrm{mM})$ but dissolved in $36 \mathrm{~mL}$ of dried 2-methylbutan-2-ol in the presence of $4 \AA$ molecular sieves $\left(100 \mathrm{~g} \mathrm{~L}^{-1}\right)$. After 5 min of sample condition under stirring at $55{ }^{\circ} \mathrm{C}$ and $200 \mathrm{rpm}, 15 \%$ (m/m of total reactants) of immobilized lipase from Candida antarctica (Novozyme $435^{\circledR}$ ) was added to the mixture. The reactions were conducted at the same conditions as described above and after $120 \mathrm{~h}$ the reaction mixtures were filtered on filter paper to separate the molecular sieve. 
Thin layer chromatography (TLC)

The solvent system tests and fractions obtained from HSCCC separations were performed by TLC on silica gel $60 \mathrm{~F}_{254}$ plates (Silicycle ${ }^{\circledR}$, Quebec, Canada) using a solvent system mix consisted of ethyl acetate/acetone/water $(5: 3: 1 ; \mathrm{v} / \mathrm{v} / \mathrm{v})$. The products were detected by spraying the Natural Products (NP) Reagent A (methanol solution of 2-aminoethyl diphenylborinate (1\%)) followed by ethanol solution of polyethylene glycol (PEG) $(5 \%)$ and revealed under $\mathrm{UV}_{365 \mathrm{~mm}}$ chamber. The absence of fatty acids was checked spraying bromocresol green $0.1 \%$ alkalized with $\mathrm{NaOH} 1 \mathrm{M}$.

\section{Solvent system tests}

The solvent systems tested for the countercurrent chromatography analyzes were composed of HEMWat (hexane/ethyl acetate/methanol/water). The test started with the analysis using the HEMWat 0 (5:5:5:5) system, varying the ratio between ethyl acetate and hexane and/or water and methanol to establish the ideal system for each sample. HEMWat in different proportions, 0:10:0:10 (No. +8), 1:9:1:9 (No. +7), 2:8:2:8 (No. +6), 3:7:3:7 (No. +5), 4:6:4:6 (No. +3), 3:7:5:5 (No. +2), 4:6:5:5 (No. +1), 5:5:5:5 (No. 0), ${ }^{34}$ were used to analyze the different polarities rutin esters. A small amount of each reaction mixtures (ca. $5 \mathrm{mg}$ ) was submitted to the test tube partitioning test with the biphasic solvent systems ( $2 \mathrm{~mL}$ ). Initially, to mix the phases, the tubes were shaken vigorously with a vortex mixer by $10 \mathrm{~s}$, and the phase separation was observed. Then, equivalent aliquots of each upper and lower phases were analyzed separately on silica gel TLC plates. The distribution of the compounds between the two phases was determined visually. Therefore, the biphasic solvent system was chosen according to the similar distribution between the phases of the target compounds (rutin ester).

\section{Purification by HSCCC}

Initially, the solvent mixture was equilibrated in a separating funnel at room temperature. The two phases were separated and degassed by sonication in Ultra Cleaner 800A (Unique ${ }^{\circledR}$, São Paulo, Brazil) for 15 min shortly before use. The column was filled with the stationary phase

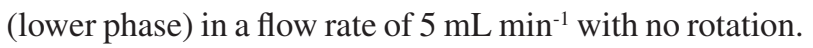
The rotation was turned on at the speed of $860 \mathrm{rpm}$, and the mobile phase (upper phase) was pumped at a flow rate of $2 \mathrm{~mL} \mathrm{~min}^{-1}$. The retention of the stationary phase, Sf, was determined by measuring the volume of stationary phase displaced until the solvent front in the equilibrium stage. Then, this volume was subtracted from the total coil volume and calculated the percentage of stationary phase that remained inside the coil. The sample (ca. 200-900 mg dissolved in $10 \mathrm{~mL}$ of the solvent system) was injected using a $10 \mathrm{~mL}$ loop, at normal phase (tail-to-head) elution mode after reaching the hydrodynamic equilibrium. Approximately 60 fractions of $4 \mathrm{~mL}$ were collected with rotation on which were assembled according to the similarity profile observed in TLC. The solvent systems used in the isolation of rutin esters (HEMWat) and the yields obtained, were established as follows: 0:10:0:10 (rutin propionate, $27.3 \mathrm{mg}$ ); 1:9:1:9 (rutin butyrate, $40.2 \mathrm{mg}$ ); 2:8:2:8 (rutin caproate, $47.4 \mathrm{mg}$ ); 3:7:3:7 (rutin caprylate $39.5 \mathrm{mg}$ ); 4:6:4:6 (rutin laurate, $51.0 \mathrm{mg}$ ); 3:7:5:5 (rutin myristate, $63.4 \mathrm{mg}$ ); 4:6:5:5 (rutin palmitate, $69.8 \mathrm{mg}$ ); 5:5:5:5 (rutin stearate, $39.4 \mathrm{mg}$ ); 4:6:5:5 (rutin oleate, $30.8 \mathrm{mg}$ ); 3:7:5:5 (rutin linolate, $49.4 \mathrm{mg}$ ).

\section{Spectroscopic data}

\section{Rutin}

${ }^{1} \mathrm{H}$ NMR (DMSO- $d_{6}$ ) $\delta 7.54$ (m, 2H, H-6'/H-2'), 6.84 (d, J $8.2 \mathrm{~Hz}, 1 \mathrm{H}, \mathrm{H}-5$ '), 6.38 (d, J $2.0 \mathrm{~Hz}, 1 \mathrm{H}, \mathrm{H}-8), 6.19$ (d, J $2.0 \mathrm{~Hz}, 1 \mathrm{H}, \mathrm{H}-6), 5.34$ (d, J 7.3 Hz, 1H, H-1"), 4.38 (s, 1H, H-1"'), 3.03-3.71 (m, sugar H), 0.99 (d, J $6.2 \mathrm{~Hz}$, 1H, H-6"'); ${ }^{13} \mathrm{C}$ NMR (DMSO- $\left.d_{6}\right) \delta 156.57$ (C-2), 133.24 (C-3), 161.18 (C-5), 98.64 (C-6), 164.19 (C-7), 93.6 (C-8), 156.41 (C-9), 103.86 (C-10), 120.98 (C-1'), 116.18 (C-2'), 144.73 (C-3'), 148.4 (C-4'), 115.16 (C-5'), 121.54 (C-6'), 101.13 (C-1"), 74.00 (C-2"), 76.34 (C-3"), 69.95 (C-4"), 75.83 (C-5"), 66.91 (C-6"), 100.68 (C-1"”), 70.28 (C-2"'), 70.46 (C-3"'), 71.75 (C-4"”), 68.15 (C-5"'), 17.62 (C-6”'); ESI-MS $m / z,[\mathrm{M}-\mathrm{H}]^{-}: 609$.

\section{Rutin propionate}

${ }^{1} \mathrm{H}$ NMR (DMSO- $\left.d_{6}\right) \delta 7.53(\mathrm{dd}, J 8.4,2.2 \mathrm{~Hz}, 1 \mathrm{H}$, H-6'), 7.50 (d, J 2.2 Hz, 1H, H-2'), 6.83 (d, J $8.4 \mathrm{~Hz}, 1 \mathrm{H}$, H-5'), 6.36 (d, J $2.0 \mathrm{~Hz}, 1 \mathrm{H}, \mathrm{H}-8), 6.17$ (d, J $2.0 \mathrm{~Hz}, 1 \mathrm{H}$, H-6), 5.42 (d, J 7.4 Hz, 1H, H-1"), 4.63 (t, J 9.8 Hz, 1H, H-4"” acylated), 4.45 (s, 1H, H-1"'), 3.14-3.67 (m, sugar $\mathrm{H}), 2.17$ (m, 2H, $\mathrm{CH}_{2} \alpha$-fatty chain), $0.98(\mathrm{t}, J 7.5 \mathrm{~Hz}, 3 \mathrm{H}$, $\mathrm{CH}_{3}$-fatty chain), 0.74 (d, J $6.3 \mathrm{~Hz}, 3 \mathrm{H}, \mathrm{CH}_{3}$-rhamnosyl); ${ }^{13} \mathrm{C}$ NMR (DMSO- $d_{6}$ ) $\delta 156.25$ (C-2/C-9), 161.06 (C-5), 98.64 (C-6), 164.36 (C-7), 93.4 (C-8), 103.59 (C-10), 120.99 (C-1'), 115.99 (C-2'), 144.65 (C-3'), 148.3 (C-4'), 115.07 (C-5'), 121.41 (C-6'), 100.82 (C-1”), 73.92 (C-2”), 76.36 (C-3”), 69.45 (C-4”), 75.33 (C-5”), 66.91 (C-6"), 100.45 (C-1"'), 70.22 (C-2"”), 67.92 (C-3"'), 73.26 (C-4"'), 65.59 (C-5"'), 16.87 (C-6"'), 173.26 (C=O fatty acid), 26.58 (C $\alpha$, fatty acid), $8.87\left(\mathrm{CH}_{3}\right.$, fatty acid); ESI-MS $m / z,[\mathrm{M}-\mathrm{H}]^{-}: 665$. 
Rutin butyrate

${ }^{1} \mathrm{H}$ NMR (DMSO- $d_{6}$ ) $\delta 7.52(\mathrm{dd}, J 8.4,2.1 \mathrm{~Hz}, 1 \mathrm{H}$, H-6'), 7.48 (d, J 2.1 Hz, 1H, H-2'), 6.82 (d, J 8.4 Hz, 1H, H-5'), 6.30 (d, J 1.8 Hz, 1H, H-8), 6.13 (d, J 1.9 Hz, 1H, H-6), 5.41 (d, J 7.4 Hz, 1H, H-1"), 4.64 (t, J $9.8 \mathrm{~Hz}$, 1H, H-4" ' acylated), 4.45 (s, 1H, H-1"'), 3.14-3.67 (m, sugar $\mathrm{H}), 2.13\left(\mathrm{~m}, 2 \mathrm{H}, \mathrm{CH}_{2} \alpha\right.$-fatty chain), $1.48(\mathrm{~m}$, $2 \mathrm{H}, \mathrm{CH}_{2} \beta$-fatty chain), $0.87\left(\mathrm{t}, J 7.4 \mathrm{~Hz}, 3 \mathrm{H}, \mathrm{CH}_{3}\right.$-fatty acid), 0.75 (d, $J 6.3 \mathrm{~Hz}, 3 \mathrm{H}, \mathrm{CH}_{3}$-rhamnosyl); ${ }^{13} \mathrm{C} \mathrm{NMR}$ $\left(\mathrm{DMSO}-d_{6}\right) \delta 156.18(\mathrm{C}-2), 98.82(\mathrm{C}-6), 93.46(\mathrm{C}-8)$, 103.33 (C-10), 120.84 (C-1'), 115.73 (C-2'), 144.87 (C-3'), 148.62 (C-4'), 114.87 (C-5'), 121.26 (C-6'), 100.77 (C-1"), 73.83 (C-2"), 76.28 (C-3"), 69.33 (C-4”), 75.15 (C-5"), 66.52 (C-6”), 100.29 (C-1"'), 70.13 (C-2"'), 67.82 (C-3"”), 73.02 (C-4"”), 65.49 (C-5"'), 16.86 (C-6"”), 172.47 (C=O fatty acid), 35.11 ( $\mathrm{C} \alpha$-fatty acid), 17.7 ( $\mathrm{C} \beta$-fatty acid), $13.19\left(\mathrm{CH}_{3}\right.$-fatty acid); ESI-MS $m / z,[\mathrm{M}-\mathrm{H}]^{-}: 679$.

\section{Rutin caproate}

${ }^{1} \mathrm{H}$ NMR (DMSO- $\left.d_{6}\right) \delta 7.50$ (m, 2H, H-6'/H-2'), 6.83 (d, J $8.3 \mathrm{~Hz}, 1 \mathrm{H}, \mathrm{H}-5$ '), 6.35 (d, J 1.9 Hz, 1H, H-8), 6.17 (d, $J 1.9 \mathrm{~Hz}, 1 \mathrm{H}, \mathrm{H}-6), 5.43$ (d, J 7.3 Hz, 1H, H-1"), 4.64 (t, $J 9.8 \mathrm{~Hz}, 1 \mathrm{H}, \mathrm{H}-4$ "' acylated), 4.45 (s, 1H, H-1" "), 3.14-3.69 $(\mathrm{m}$, sugar $\mathrm{H}), 2.14\left(\mathrm{~m}, 2 \mathrm{H}, \mathrm{CH}_{2} \alpha\right.$-fatty chain), $1.45(\mathrm{~m}, 2 \mathrm{H}$, $\mathrm{CH}_{2} \beta$-fatty chain), $1.26\left(\mathrm{~m}, 4 \mathrm{H}, \mathrm{CH}_{2}\right.$-fatty chain), 0.87 (t, $J 7.0 \mathrm{~Hz}, 3 \mathrm{H}, \mathrm{CH}_{3}$ fatty acid), $0.74\left(\mathrm{~d}, J 6.2 \mathrm{~Hz}, 3 \mathrm{H}, \mathrm{CH}_{3}\right.$ rhamnosyl); ${ }^{13} \mathrm{C}$ NMR (DMSO- $d_{6}$ ) $\delta 156.44$ (C-2), 161.2 (C-5), 98.74 (C-6), 164.36 (C-7), 93.48 (C-8), 156.41 (C-9), 103.73 (C-10), 121.06 (C-1'), 116.05 (C-2'), 144.81 (C-3'), 148.37 (C-4'), 115.16 (C-5'), 121.49 (C-6'), 100.89 (C-1"), 74.04 (C-2”), 76.49 (C-3”), 69.54 (C-4”), 75.45 (C-5”), 66.76 (C-6"), 100.46 (C-1"'), 70.37 (C-2"'), 68.08 (C-3"'), 73.28 (C-4"”), 65.68 (C-5”"), 17.01 (C-1"”), 172.66 (C=O fatty acid), 33.33 ( $\mathrm{C} \alpha$-fatty acid), 24.09 ( $\mathrm{C} \beta$, fatty acid), $30.54\left(\mathrm{CH}_{2}\right.$-fatty chain), $21.78\left(\mathrm{CH}_{2}\right.$-fatty chain $), 13.8\left(\mathrm{CH}_{3}-\right.$ fatty acid); ESI-MS $m / z,[\mathrm{M}-\mathrm{H}]^{-}: 707$.

\section{Rutin caprylate}

${ }^{1} \mathrm{H}$ NMR (DMSO- $\left.d_{6}\right) \delta 7.51$ (m, 2H, H-6'/H-2') 6.83 (d, $J 8.3 \mathrm{~Hz}, 1 \mathrm{H}, \mathrm{H}-5$ '), 6.35 (d, $J 1.9 \mathrm{~Hz}, 1 \mathrm{H}, \mathrm{H}-8), 6.17$ (d, J $2.0 \mathrm{~Hz}, 1 \mathrm{H}, \mathrm{H}-6), 5.43$ (d, J 7.4 Hz, 1H, H-1"), 4.64 (t, $J 9.8 \mathrm{~Hz}, 1 \mathrm{H}, \mathrm{H}-4$ "' acylated), 4.45 (s, 1H, H-1"'), 3.13-3.69 (m, sugar $\mathrm{H}), 2.15\left(\mathrm{~m}, 2 \mathrm{H}, \mathrm{CH}_{2} \alpha\right.$-fatty chain), 1.46 (m, $2 \mathrm{H}, \mathrm{CH}_{2} \beta$-fatty chain), 1.27 (m, $8 \mathrm{H}, \mathrm{CH}_{2}$-fatty chain), 0.86 (t, $J 6.9 \mathrm{~Hz}, 3 \mathrm{H}, \mathrm{CH}_{3}$-fatty acid), 0.75 (d, $J 6.2 \mathrm{~Hz}, 3 \mathrm{H}, \mathrm{CH}_{3}$-rhamnosyl); ${ }^{13} \mathrm{C}$ NMR (DMSO- $d_{6}$ ) $\delta 156.54$ (C-2), 161.26 (C-5), 98.57 (C-6), 164.32 (C-7), 93.33 (C-8), 156.38 (C-9), 103.73 (C-10), 121.05 (C-1'), 116.00 (C-2'), 144.83 (C-3'), 148.42 (C-4'), 115.00 (C-5'), 121.3 (C-6'), 100.75 (C-1"), 73.91 (C-2"), 76.33 (C-3"), 69.43 (C-4"), 75.32 (C-5"), 66.62 (C-6"), 100.32 (C-1"”),
70.24 (C-2"”), 67.95 (C-3"'), 73.15 (C-4"”), 65.57 (C-5"'), 16.91 (C-6"'), 172.59 (C=O fatty acid), 33.27 (C $\alpha$, fatty acid), 24.32 (Cß-fatty acid), 21.91-30.97 $\left(\mathrm{CH}_{2}\right.$-fatty chain), $13.78\left(\mathrm{CH}_{3}\right.$-fatty acid); ESI-MS $m / z,[\mathrm{M}-\mathrm{H}]^{-}: 725$.

\section{Rutin laurate}

${ }^{1} \mathrm{H}$ NMR (DMSO- $\left.d_{6}\right) \delta 7.50$ (m, 2H, H-6'/H-2'), 6.83 (d, J 8.2 Hz, 1H, H-5'), 6.34 (s, 1H, H-8), 6.17 (s, 1H, H-6), 5.42 (d, J 7.1 Hz, 1H, H-1"), 4.64 (t, $J 9.7 \mathrm{~Hz}, 1 \mathrm{H}, \mathrm{H}-4$ "' acylated), 4.45 (s, 1H, H-1"'), 3.14-3.69 (m, sugar H), $2.14\left(\mathrm{~m}, 2 \mathrm{H}, \mathrm{CH}_{2} \alpha\right.$-fatty chain), $1.44\left(\mathrm{~m}, 2 \mathrm{H}, \mathrm{CH}_{2} \beta\right.$-fatty chain), 1.23 (s, $16 \mathrm{H}, \mathrm{CH}_{2}$-fatty chain), 0.84 (t, $J 6.7 \mathrm{~Hz}, 3 \mathrm{H}$, $\mathrm{CH}_{3}$-fatty acid), 0.75 (d, $J 6.2 \mathrm{~Hz}, 3 \mathrm{H}, \mathrm{CH}_{3}$-rhamnosyl); ${ }^{13} \mathrm{C}$ NMR (DMSO- $d_{6}$ ) $\delta 156.42$ (C-2), 161.21 (C-5), 98.69 (C-6), 164.47 (C-7), 93.43 (C-8), 156.38 (C-9), 103.69 (C-10), 121.02 (C-1'), 116.08 (C-2'), 144.81 (C-3'), 148.4 (C-4'), 115.08 (C-5'), 121.39 (C-6'), 100.87 (C-1'), 73.97 (C-2"), 76.42 (C-3"), 69.48 (C-4”), 75.41 (C-5"), 66.74 (C-6"), 100.42 (C-1"'), 70.31 (C-2"'), 68.03 (C-3"'), 73.23 (C-4"'), 65.64 (C-5"'), 17.01 (C-6"'), 172.61 (C=O fatty acid), 33.36 ( $\mathrm{C} \alpha$, fatty acid), 24.39 (C $\beta$-fatty acid), 21.99-31.22 ( $\mathrm{CH}_{2}$-fatty chain), $13.87\left(\mathrm{CH}_{3}\right.$-fatty acid $)$; ESI-MS $m / z,[\mathrm{M}-\mathrm{H}]^{-}: 791$.

\section{Rutin myristate}

${ }^{1} \mathrm{H}$ NMR (DMSO- $\left.d_{6}\right) \delta 7.50$ (m, 2H, H-6'/H-2'), 6.83 (d, J 8.2 Hz, 1H, H-5'), 6.34 (d, J 1.9 Hz, 1H, H-8), 6.17 (d, J $1.9 \mathrm{~Hz}, 1 \mathrm{H}, \mathrm{H}-6), 5.42$ (d, J 7.1 Hz, 1H, H-1"), 4.63 (t, $J 9.7 \mathrm{~Hz}, 1 \mathrm{H}, \mathrm{H}-4$ "' acylated), 4.45 (s, 1H, H-1"'), 3.15-3.68 (m, sugar $\mathrm{H}), 2.13(\mathrm{~m}, J 6.9,3.2 \mathrm{~Hz}, 2 \mathrm{H}$, $\mathrm{CH}_{2} \alpha$-fatty chain), $1.44\left(\mathrm{~m}, J 2.7 \mathrm{~Hz}, 2 \mathrm{H}, \mathrm{CH}_{2} \beta\right.$-fatty chain), 1.22 (s, $17 \mathrm{H}, \mathrm{CH}_{2}$-fatty chain), $0.83(\mathrm{t}, J 6.7 \mathrm{~Hz}, 2 \mathrm{H}$, $\mathrm{CH}_{3}$-fatty acid), 0.74 (d, $J 6.2 \mathrm{~Hz}, 2 \mathrm{H}, \mathrm{CH}_{3}$-rhamnosyl); ${ }^{13} \mathrm{C}$ NMR (DMSO- $d_{6}$ ) $\delta 156.64$ (C-2), 161.39 (C-5), 98.76 (C-6), 164.46 (C-7), 93.56 (C-8), 156.53 (C-9), 103.89 (C-10), 121.18 (C-1'), 116.24 (C-2'), 144.97 (C-3'), 148.53 (C-4'), 115.23 (C-5'), 121.55 (C-6'), 100.95 (C-1'), 74.13 (C-2”), 76.56 (C-3"), 69.59 (C-4”), 75.48 (C-5”), 66.76 (C-6"), 100.55 (C-1"”), 70.41 (C-2"'), 68.18 (C-3"'), 73.36 (C-4"'), 65.74 (C-5"'), 17.11 (C-6"'), 172.85 (C=O fatty acid), $33.46(\mathrm{C} \alpha$, fatty acid), 24.5 (C $\beta$-fatty acid), 22.16-31.34 ( $\mathrm{CH}_{2}$-fatty chain), $14.00\left(\mathrm{CH}_{3}\right.$-fatty acid $)$; ESI-MS $m / z,[\mathrm{M}-\mathrm{H}]^{-}: 819$.

\section{Rutin palmitate}

${ }^{1} \mathrm{H}$ NMR (DMSO- $\left.d_{6}\right) \delta 7.50$ (m, 2H, H-6'/H-2'), 6.83 (d, J $8.3 \mathrm{~Hz}, 1 \mathrm{H}, \mathrm{H}-5$ "), 6.34 (d, J $1.9 \mathrm{~Hz}, 1 \mathrm{H}, \mathrm{H}-8), 6.17$ (d, J $2.0 \mathrm{~Hz}, 1 \mathrm{H}, \mathrm{H}-6), 5.42$ (d, J 7.3 Hz, 1H, H-1"), 4.64 (t, J $9.8 \mathrm{~Hz}, 1 \mathrm{H}, \mathrm{H}-4$ "' acylated), 4.45 (s, 1H, H-1"'), 3.15-3.68 (m, sugar $\mathrm{H}), 2.14$ (m, $2 \mathrm{H}, \mathrm{CH}_{2} \alpha$-fatty chain), $1.44\left(\mathrm{~m}, 2 \mathrm{H}, \mathrm{CH}_{2} \beta\right.$-fatty chain), $1.19\left(\mathrm{~m}, 22 \mathrm{H}, \mathrm{CH}_{2}\right.$-fatty 
chain), 0.84 (t, J $6.9 \mathrm{~Hz}, 3 \mathrm{H}, \mathrm{CH}_{3}$-fatty acid), 0.75 (d, $J 6.2 \mathrm{~Hz}, 3 \mathrm{H}, \mathrm{CH}_{3}$-rhamnosyl); ${ }^{13} \mathrm{C}$ NMR (DMSO- $d_{6}$ ) $\delta 156.43$ (C-2), 161.15 (C-5), 98.71 (C-6), 164.28 (C-7), 93.45 (C-8), 156.33 (C-9), 103.77 (C-10), 121.07 (C-1'), 116.16 (C-2'), 144.82 (C-3'), 148.37 (C-4'), 115.13 (C-5'), 121.48 (C-6'), 100.91 (C-1"), 74.04 (C-2"), 76.42 (C-3"), 69.52 (C-4"), 75.45 (C-5"), 66.74 (C-6"), 100.49 (C-1"'), 70.37 (C-2"'), 68.09 (C-3"'), 73.3 (C-4"'), 65.69 (C-5"'), 17.03 (C-6"'), 172.58 (C=O fatty acid), 33.42 (Co, fatty acid), 24.42 (C $\beta$ - fatty acid), 22.04-31.33 $\left(\mathrm{CH}_{2}\right.$, fatty chain), $13.9\left(\mathrm{CH}_{3}\right.$, fatty acid); ESI-MS $m / z,[\mathrm{M}-\mathrm{H}]^{-}: 847$.

\section{Rutin stearate}

${ }^{1} \mathrm{H}$ NMR (DMSO- $\left.d_{6}\right) \delta 7.51$ (m, 2H, H-6'/H-2'), 6.83 (d, J 8.1 Hz, 1H, H-5'), 6.35 (d, J 1.9 Hz, 1H, H-8), 6.18 (d, $J 1.8 \mathrm{~Hz}, 1 \mathrm{H}, \mathrm{H}-6), 5.43$ (d, J 7.3 Hz, 1H, H-1"), 4.64 (t, J $9.8 \mathrm{~Hz}, 1 \mathrm{H}, \mathrm{H}-4$ "' acylated), 4.45 (s, 1H, H-1"'), 3.14-3.69 (m, sugar $\mathrm{H}), 2.14$ (m, $2 \mathrm{H}, \mathrm{CH}_{2} \alpha$-fatty chain), $1.45\left(\mathrm{~m}, 2 \mathrm{H}, \mathrm{CH}_{2} \beta\right.$-fatty chain), $1.22\left(\mathrm{~m}, 28 \mathrm{H}, \mathrm{CH}_{2}\right.$-fatty chain), 0.84 (t, $J 6.9 \mathrm{~Hz}, 3 \mathrm{H}, \mathrm{CH}_{3}$-fatty acid), 0.75 (d, $J 6.2 \mathrm{~Hz}, 3 \mathrm{H}, \mathrm{CH}_{3}$-rhamnosyl); ${ }^{13} \mathrm{C}$ NMR (DMSO- $d_{6}$ ) $\delta 156.49(\mathrm{C}-2), 161.27$ (C-5), $98.6(\mathrm{C}-6), 164.3(\mathrm{C}-7), 93.39$ (C-8), 156.4 (C-9), 103.79 (C-10), 121.06 (C-1'), 116.12 (C-2'), 144.81 (C-3'), 148.36 (C-4'), 115.08 (C-5'), 121.39 (C-6'), 100.87 (C-1"), 73.98 (C-2"), 76.42 (C-3"), 69.48 (C-4"), 75.43 (C-5"), 66.6 (C-6"), 100.42 (C-1"”), 70.31 (C-2"'), 68.06 (C-3"'), 73.25 (C-4"'), 65.63 (C-5"”), 16.99 (C-6"'), 172.6 (C=O fatty acid), 33.25 ( $\mathrm{C} \alpha$, fatty acid), 24.39 (Cß-fatty acid), 22.06-31.12 ( $\mathrm{CH}_{2}$-fatty chain), 13.85 $\left(\mathrm{CH}_{3}\right.$-fatty acid); ESI-MS $\mathrm{m} / z,[\mathrm{M}-\mathrm{H}]^{-}: 875$.

\section{Rutin oleate}

${ }^{1} \mathrm{H}$ NMR (DMSO- $\left.d_{6}\right) \delta 7.51$ (m, 2H, H-6'/H-2'), 6.83 (d, J $8.1 \mathrm{~Hz}, 1 \mathrm{H}, \mathrm{H}-5$ ') 6.36 (s, 1H, H-8), 6.17 (s, 1H, H-6), 5.42 (d, J 6.5 Hz, 1H, H-1"), 5.32 (s, $2 \mathrm{H}, \mathrm{CH}_{2 \text { olefinic- }}$ fatty chain), 4.64 (t, $J 9.7 \mathrm{~Hz}, 1 \mathrm{H}, \mathrm{H}-4$ "' acylated), 4.45 (s, $1 \mathrm{H}, \mathrm{H}-1$ "'), 2.15 (m, 2H, $\mathrm{CH}_{2} \alpha$-fatty chain), 1.97 (m, $4 \mathrm{H}, \mathrm{CH}_{2}$-fatty chain), 1.45 ( $\mathrm{m}, 3 \mathrm{H}, \mathrm{CH}_{2} \beta$-fatty chain), 1.23 $\left(\mathrm{m}, J 15.2 \mathrm{~Hz}, 20 \mathrm{H}, \mathrm{CH}_{2}\right.$-fatty chain), $0.84(\mathrm{t}, J 6.6 \mathrm{~Hz}, 3 \mathrm{H}$, $\mathrm{CH}_{3}$-fatty acid), 0.75 (d, J $6.0 \mathrm{~Hz}, 3 \mathrm{H}, \mathrm{CH}_{3}$-rhamnosyl); ${ }^{13} \mathrm{C}$ NMR (DMSO- $\left.d_{6}\right) \delta 156.44(\mathrm{C}-2), 161.12(\mathrm{C}-5), 98.66$ (C-6), 164.21 (C-7), 93.42 (C-8), 156.36 (C-9), 103.75 (C-10), 116.15 (C-2'), $144.77\left(\mathrm{C}-3^{\prime}\right), 148.35$ (C-4'), 115.06 (C-5'), 121.41 (C-6'), 100.88 (C-1"), 74.02 (C-2”), 76.44 (C-3"), 69.51 (C-4"), 75.47 (C-5"), 66.75 (C-6"), 100.43 (C-1"”), 70.35 (C-2"'), 68.07 (C-3"'), 73.22 (C-4"”), 65.67 (C-5"'), 17.04 (C-6"'), 172.56 (C=O fatty acid), $33.41(\mathrm{C} \alpha$, fatty acid), 24.56 ( $\mathrm{C} \beta$-fatty acid), $28.68\left(\mathrm{CH}_{2}\right.$-fatty chain), $26.52\left(\mathrm{CH}_{2 \text { allylic }}\right.$-fatty chain), $129.58\left(\mathrm{CH}_{2 \text { olefinic }}\right.$-fatty chain $)$, $31.19\left(\mathrm{CH}_{2} \omega_{3}\right.$-fatty chain), $22.04\left(\mathrm{CH}_{2} \omega_{2}\right.$-fatty chain $)$, $13.89\left(\mathrm{CH}_{3}\right.$-fatty acid); ESI-MS $m / z,[\mathrm{M}-\mathrm{H}]^{-}: 873$.
Rutin linolate

${ }^{1} \mathrm{H}$ NMR (DMSO- $\left.d_{6}\right) \delta 7.51$ (m, 2H, H-6'/H-2'), 6.83 (d, J 8.1 Hz, 1H, H-5'), 6.34 (d, J 1.9 Hz, 1H, H-8), 6.17 (d, J $1.9 \mathrm{~Hz}, 1 \mathrm{H}, \mathrm{H}-6), 5.42$ (d, J $7.3 \mathrm{~Hz}, 1 \mathrm{H}, \mathrm{H}-1$ ”), $5.31\left(\mathrm{~m}, 4 \mathrm{H}, \mathrm{CH}_{2 \text { olefinic }}\right.$-fatty chain), $4.64(\mathrm{t}, J 9.8 \mathrm{~Hz}, 1 \mathrm{H}$, H-4"' cylated), 4.45 (s, 1H, H-1"'), 2.72 (t, J $6.5 \mathrm{~Hz}, 2 \mathrm{H}$, $\mathrm{CH}_{2}$ bis-allylic-fatty chain), $2.14\left(\mathrm{~m}, 2 \mathrm{H}, \mathrm{CH}_{2} \alpha\right.$-fatty chain), $2.01\left(\mathrm{~m}, 4 \mathrm{H}, \mathrm{CH}_{2}\right.$ allylic -fatty chain), $1.45\left(\mathrm{~m}, 2 \mathrm{H}, \mathrm{CH}_{2} \beta\right.$-fatty chain), 1.28 (m, 14H, $\mathrm{CH}_{2}$-fatty chain), $0.84(\mathrm{t}, J 6.9 \mathrm{~Hz}$, $3 \mathrm{H}, \mathrm{CH}_{3}$-fatty acid), 0.75 (d, J6.2 Hz, 3H, $\mathrm{CH}_{3}$-rhamnosyl); ${ }^{13} \mathrm{C}$ NMR (DMSO- $\left.d_{6}\right) \delta 156.46(\mathrm{C}-2), 161.22(\mathrm{C}-5), 98.62$ (C-6), 164.36 (C-7), 93.38 (C-8), 156.38 (C-9), 103.73 (C-10), 121.02 (C-1'), 116.08 (C-2'), 144.8 (C-3'), 148.41 (C-4'), 115.04 (C-5'), 121.37 (C-6'), 100.86 (C-1'”), 73.95 (C-2"), 76.37 (C-3"), 75.38 (C-5"), 66.63 (C-6"), 100.39 (C-1"'), 70.28 (C-2"'), 68.02 (C-3"'), 73.22 (C-4"'), 65.59 (C-5"'), 17.01 (C-6"'), 172.6 (C=O fatty acid), 33.36 (C $\mathrm{C}$, fatty acid), 24.36 (C $\beta$-fatty acid), $28.39\left(\mathrm{CH}_{2}\right.$ - fatty chain), $26.51\left(\mathrm{CH}_{2 \text { allylic }}\right.$-fatty chain $), 127.65\left(\mathrm{CH}_{2 \text { olefinic }}\right.$-fatty chain $)$, $25.1\left(\mathrm{CH}_{2 \text { bis-allylic }}\right.$-fatty chain $), 129.6\left(\mathrm{CH}_{2 \text { olefinic }}\right.$-fatty chain $)$, $30.81\left(\mathrm{CH}_{2} \omega_{3}\right.$-fatty chain), $21.97\left(\mathrm{CH}_{2} \omega_{2}\right.$-fatty chain), $13.85\left(\mathrm{CH}_{3}\right.$-rhamnosyl); ESI-MS $\mathrm{m} / \mathrm{z},[\mathrm{M}-\mathrm{H}]^{-}: 871$.

\section{Results and Discussion}

Enzymatic synthesis of rutin fatty acid esters

The synthesis of rutin esters were performed in 2-methylbutan-2-ol. This solvent was chosen based on solubility tests in which it presented the higher solubility capacity $(60.03 \pm 0.40 \mathrm{mM})$ compared to other solvents previously used in esterification reactions, such as acetone $(13.50 \pm 0.34 \mathrm{mM})$ and acetonitrile $(0.50 \pm 0.01 \mathrm{mM}){ }^{35}$ Furthermore, this solvent presents low toxicity and does not promote enzymatic denaturation. ${ }^{36}$

The conversions obtained in this work with 2-methylbutan-2-ol as reaction solvent ranged from 16 to $40 \%$ (Figure 1), while in a previous study ${ }^{14}$ there were observed conversion rates ranging from 27 to $62 \%$ for esterification reactions with different fatty acids. As can be seen, our conversions were slightly lower, but this fact can be justified by the difference in the conditions established for the esterification process. Among them, it is important to highlight the substrates concentration that was 4-fold higher than that evaluated in this work. In addition to that, the enzymatic concentration ranged from 10 to $20 \%$ based on total substrate mass $\left(10 \mathrm{~g} \mathrm{~L}^{-1}\right)$ while we fixed $15 \% \mathrm{~m} / \mathrm{m}$ of total reactants, as well as the reaction time was longer (up to $168 \mathrm{~h}$ versus $120 \mathrm{~h}$ ) and the amount of molecular sieve was higher $\left(150 \mathrm{~g} \mathrm{~L}^{-1}\right)$ than it used in our study. ${ }^{14}$ Besides that, another studies ${ }^{10,11}$ using fatty acids with different 
chain sizes reached ester conversions ranging from 40 to $76 \%$. However, lower concentration of the substrates (molar ratio of $1: 1$ ), higher than $100 \% \mathrm{~m} / \mathrm{m}$ of the enzyme (based on total substrate mass) and $150 \mathrm{~g} \mathrm{~L}^{-1}$ of molecular sieves were applied. In a previous research, ${ }^{15}$ in which there were used a minor concentration of the substrates and about 3 -fold more enzyme compared to this work and $100 \mathrm{~g} \mathrm{~L}^{-1}$ of molecular sieves, it was observed a conversion of $45 \%$ for the rutin ester with oleic acid in $96 \mathrm{~h}$ reaction-time.

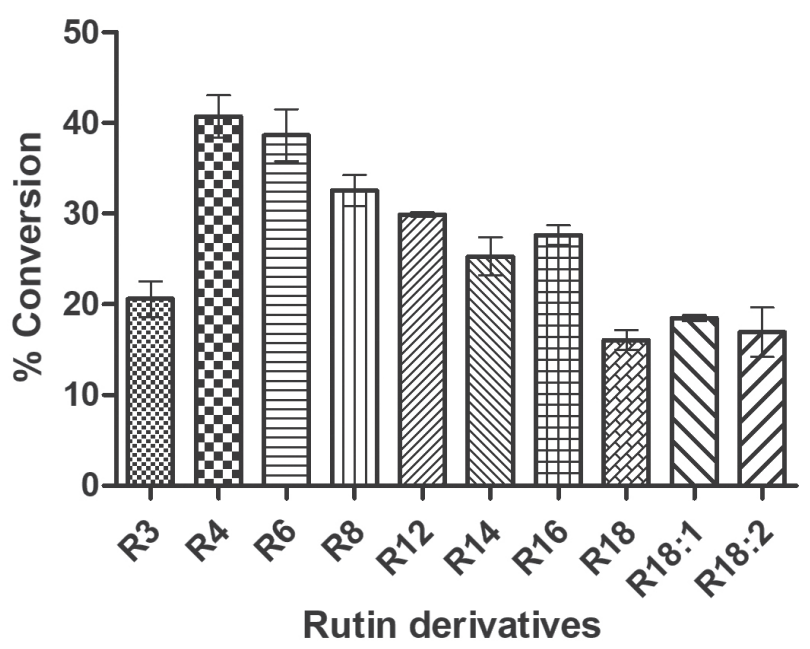

Figure 1. Difference on fatty acid chain length on conversions to rutin derivatives at $120 \mathrm{~h}$. Reaction conditions: rutin $\left(13.65 \mathrm{mmol} \mathrm{L}^{-1}\right)$; fatty acids $\left(68.25 \mathrm{mmol} \mathrm{L}^{-1}\right) ; 4 \AA$ molecular sieves $\left(100 \mathrm{~g} \mathrm{~L}^{-1}\right) ; 15 \%$ (m/m of total reactants) of Novozyme $435^{\circledR}$ in $3 \mathrm{~mL} 2$-methylbutan-2-ol at $55^{\circ} \mathrm{C}$ and $200 \mathrm{rpm}$. Results are expressed as mean \pm SEM (standard error of the mean). R3: rutin propionate; R4: rutin butyrate; R6: rutin caproate; R8: rutin caprylate; R12: rutin laurate; R14: rutin myristate; R16: rutin palmitate; R18: rutin stearate; R18:1: rutin oleate; R18:2: rutin linolate.

Despite the difference concerning the conversions observed in this work compared to those previous found in literature, the influence on the type and size of the fatty acid chain on the esterification of rutin seems to present a similar behavior, as will be discussed. ${ }^{14}$ The highest conversion $(\geq 30 \%)$ was reached for short chain fatty acids (R4-R12) while with rise carbon number of the fatty acids, the conversion tends to decrease (Figure 1). These observations may be attributed to the structure of the fatty acid in the binding site of $C$. antarctica that is elliptical and narrow with $9.5 \times 4.5 \AA$. The 13 carbons fatty acid is the longest one that completely fit in the fatty acid binding site of the enzyme. Thus, the increase in the number of carbons can leads to low reaction efficiency due to steric hindrance. ${ }^{4}$

\section{Rutin esters isolation by HSCCC}

The choice for the most suitable solvent system is essential for a successful HSCCC separation, and the distribution coefficient $\left(\mathrm{K}_{\mathrm{D}}\right)$ is the most significant parameter for this selection. ${ }^{37}$ The $\mathrm{K}_{\mathrm{D}}$ is the ratio of the compound between the stationary phase and the mobile phase mutually equilibrated. A smaller $K_{D}$ elutes the compound near to the solvent front presenting a lower resolution, while a larger $\mathrm{K}_{\mathrm{D}}$ can lead to a better resolution but ample and more dilute peaks caused by a longer elution time. ${ }^{38}$

The samples are basically composed by fatty acid, rutin ester and rutin. So, the biphasic solvent system was chosen since rutin ester was distributed between the upper/ lower phases $\left(0.5<\mathrm{K}_{\mathrm{D}}<2.0\right)$, and rutin concentrated in the aqueous phase $\left(\mathrm{K}_{\mathrm{D}}>2.0\right) .^{33}$ The fatty acid would have no retention $\left(K_{D}<0.5\right)$ in this system (Figure S11, Supplementary Information section).

Initially, the ideal system for the rutin propionate (R3) was investigated starting with the HEMWat solvent system family $^{34}$ at a ratio of 5:5:5:5 (No. 0), which was adjusted according to obtained results and it was identified the HEMWat 0:10:0:10 (No. +8) for the most polar rutin ester tested. Knowing that the other esters showed a gradual reduction in polarity, it was decided to test solvent systems immediately above 0:10:0:10 (No. +8) that demonstrate a decrease in the ratio of ethyl acetate to hexane and/or water to methanol. In this way, a correlation was established between the decrease in the polarity of the esters and the systems immediately previous (less polar) that was chosen for the more polar ester (R3), such as 1:9:1:9 (No. +7) for rutin butyrate (R4), 2:8:2:8 (No. +6) for rutin caproate (R6), 3:7:3:7 (No. +5) for rutin caprylate (R8), 4:6:4:6 $($ No. +3$)$ for rutin laurate (R12), 3:7:5:5 (No. +2) for rutin myristate (R14), 4:6:5:5 (No. +1) for rutin palmitate (R16), and 5:5:5:5 (No. 0) for rutin stearate (R18). The rutin oleate (R18:1) and rutin linolate (R18:2), due to their similar structures with R18, were tested first with system HEMWat 5:5:5:5 (No. 0) adjustments were made, and systems HEMWat 4:6:5:5 (No. +1) and 3:7:5:5 (No. +2) were chosen respectively (Table 1 ).

In the CCC, the lower or upper phase can be used as the mobile phase, allowing the versatility of the technique to isolate natural products, such as flavonoid. ${ }^{33,39,40}$ Considering the chemical profiles of the samples, the upper phase was chosen as the mobile phase thus allowing the rapid drying of the pooled fractions, and avoiding the degradation of the products (rutin esters) in the aqueous phase.

Before the injection of approximately $200-900 \mathrm{mg}$ of each reaction mixture $\left(\mathrm{M}_{\text {mixture }}\right)$ diluted in $10 \mathrm{~mL}$ of equal parts of biphasic solvent, the stationary phase retention $\left(\mathrm{S}_{\mathrm{f}}\right)$ ranged from 75 to $83 \%$, in accordance to the different systems resulting in a successful separation. Fractions were combined according to the TLC profile in each analysis and the total mass of ester $\left(\mathrm{M}_{\text {ester }}\right)$ produced in each 
Table 1. Data related to isolation of rutin esters in HSCCC

\begin{tabular}{lcccccccccc}
\hline $\mathrm{R}$ & 3 & 4 & 6 & 8 & 12 & 14 & 16 & 18 & $18: 1$ & $18: 2$ \\
\hline HEMWat & $0: 10: 0: 10$ & $1: 9: 1: 9$ & $2: 8: 2: 8$ & $3: 7: 3: 7$ & $4: 6: 4: 6$ & $3: 7: 5: 5$ & $4: 6: 5: 5$ & $5: 5: 5: 5$ & $4: 6: 5: 5$ & $3: 7: 5: 5$ \\
$\mathrm{Sf} / \%$ & 75 & 77 & 79 & 79 & 79 & 76 & 81 & 83 & 80 & 82 \\
$\mathrm{M}_{\text {mixture }} / \mathrm{mg}$ & 215.0 & 163.0 & 317.0 & 374.0 & 681.0 & 668.0 & 728.0 & 952.0 & 853.0 & 687.0 \\
Fraction & $20-34$ & $22-40$ & $20-37$ & $21-40$ & $32-60$ & $29-50$ & $28-55$ & $28-60$ & $28-60$ & $26-41$ \\
$\mathrm{M}_{\text {ester }} / \mathrm{mg}$ & 27.3 & 40.2 & 47.4 & 39.5 & 51.0 & 63.4 & 69.8 & 39.4 & 30.8 & 49.4 \\
Purity / \% & 97 & 89 & 98 & 99 & $>99$ & $>99$ & 98 & 93 & 96 & $>99$ \\
\hline
\end{tabular}

HEMWat: hexane/ethyl acetate/methanol/water; Sf: stationary phase retention; $\mathbf{M}_{\text {mixture }}$ : mass of biocatalysis reaction mixture; $\mathbf{M}_{\text {ester }}:$ mass of ester isolated by HSCCC from each biocatalysis reaction; R3: rutin propionate; R4: rutin butyrate; R6: rutin caproate; R8: rutin caprylate; R12: rutin laurate; R14: rutin myristate; R16: rutin palmitate; R18: rutin stearate; R18:1: rutin oleate; R18:2: rutin linolate.

esterification reaction was recovery. All rutin esters were isolated in one-step isocratic elution and the yields (mg) are presented in Table 1.

The experimental results showed that the rutin esters R3, R6, R8, R12, R14, R16, R18:1 and R18:2 were successfully isolated, with purity above $96 \%$ calculated by HPLC-DAD. In the other hand, the rutin butyrate and rutin stearate presented purity below $94 \%$ suggesting that the used systems could be adjusted to achieve even better results (Table 1). However, the quick choice of the biphasic solvent system and separation allowed a rapid recovery of the esters and prevented their degradation. Furthermore, it was possible to recover rutin and the residual fatty acid for reuse in other reaction processes.

Previous studies described the isolation of rutin esters employing column chromatography using silica gel, ${ }^{14-17,19-21}$ semi-preparative HPLC, ${ }^{12,13}$ liquid-liquid partition ${ }^{10,11,29,30}$ or preparative TLC. ${ }^{22,31}$ However, as it is known, these techniques present significant disadvantages compared to HSCCC, such as impregnation and loss of silica sample, the difficulty of substrate recovery, limitation of the amount of sample applied by analysis, use of many washing steps. Moreover, in most of the studies described, the authors do not give information concerning the efficiency of the isolation, as is being discussed here.

Ultra-performance liquid chromatography coupled to diode array and mass spectrometry detectors (UHPLC-DAD-MS/ MS)

All reaction mixtures and the purified esters obtained by HSCCC were analyzed by UHPLC-DAD-MS/MS. Both evaluations allowed the identification of the molecular ion of the products (Table 1 ) and the major fragments $m / z 609$, 463 and 301 in the negative ionization mode (Figure 2).

Through which, it was possible to confirm the esterification of rutin with the different chains of fatty acids (Table 2). The derivatives presented from 90 to
$>99 \%$ of relative purity, determined by HPLC-DAD (Table 1).

Other studies have already described the isolation of some of these esters by other methodologies, but most of them do not show the percentage of purity achieved. The only data available is concerning the purity of rutin laurate, palmitate, oleate and linolate esters, that are $>98 \%$ for rutin laurate; $>94 \%$ or $>97 \%$ for rutin palmitate,,$^{10,29}$ and $>97 \%$ for rutin oleate and linolate. ${ }^{30}$ These results agree with those found in the present study.

\section{Structural elucidation of the generated compounds}

Despite the results obtained by UHPLC-DAD-MS/ MS have been confirmed the esterification of rutin by the different acids in a single hydroxyl group, the analysis was not accurate to assert the position of the esterification in the molecule.

The specific local of esterification of rutin biocatalyzed by lipase B of Candida antarctica (Novozyme $435^{\circledR}$ ) has been widely discussed in literature. Considering that there are different findings in the literature, it is open to discussion whether it occurs in the 4"'-OH position of the rhamnose portion ${ }^{10-17}$ or at the 3 "- $-\mathrm{OH}$ position of the glucose portion of the molecule, ${ }^{18,19}$ or in both; ${ }^{20}$ it is also possible in positions 4"-OH (glucose) and 4"'-OH (rhamonse). ${ }^{21}$ Among the rutin esters studied in this work, only laurate, palmitate, stearate and oleate derivatives present previous structural elucidation described in the literature, ${ }^{10-17}$ lacking this information.

From this perspective, it is supposed that the molecules produced in the present work may also present an esterification profile similar to those already described, aspect that was confirmed by the NMR analysis.

The monodimensional and bidimensional NMR analyses allow to confirm the esterification of the compounds in the 4" "position showing HMBC correlations with C-6"', C-3"', C-5"' and C=O of fatty acid (Figure 3). 


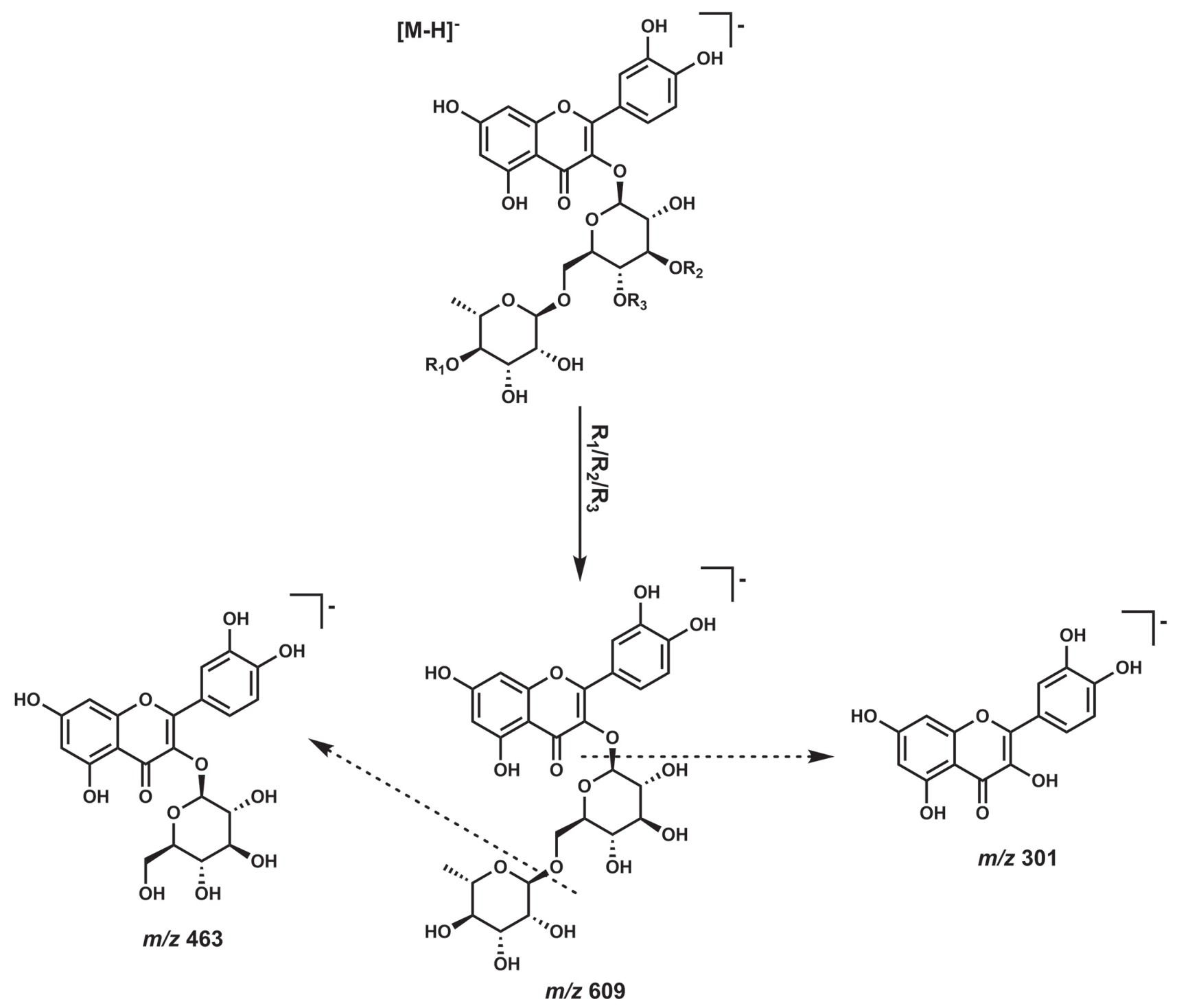

Figure 2. Deprotonated ions $[\mathrm{M}-\mathrm{H}]^{-}$of rutin derivatives obtained by the esterification reactions catalyzed by Novozyme $435^{\circledR}$ detected by mass spectrometry (ESI-negative ionization mode). In the figure, there are represented all possible esterification positions described in the literature ${ }^{10-21}\left(\mathrm{R}_{1} / \mathrm{R}_{2} / \mathrm{R}_{3}\right)$.

Table 2. Deprotonated ions $[\mathrm{M}-\mathrm{H}]^{-}$of rutin derivatives obtained by the esterification reactions catalyzed by Novozyme $435^{\circledR}$ detected by mass spectrometry (ESI-negative ionization mode)

\begin{tabular}{lcc}
\hline Product & $\mathrm{R}_{1} / \mathrm{R}_{2} / \mathrm{R}_{3}$ & {$[\mathrm{M}-\mathrm{H}]^{-}$} \\
\hline Rutin propionate & $\mathrm{C}_{3} \mathrm{H}_{6} \mathrm{O}_{2}$ & 665 \\
Rutin butyrate & $\mathrm{C}_{4} \mathrm{H}_{8} \mathrm{O}_{2}$ & 679 \\
Rutin caproate & $\mathrm{C}_{6} \mathrm{H}_{12} \mathrm{O}_{2}$ & 707 \\
Rutin caprylate & $\mathrm{C}_{8} \mathrm{H}_{16} \mathrm{O}_{2}$ & 725 \\
Rutin laurate & $\mathrm{C}_{12} \mathrm{H}_{24} \mathrm{O}_{2}$ & 791 \\
Rutin myristate & $\mathrm{C}_{14} \mathrm{H}_{28} \mathrm{O}_{2}$ & 819 \\
Rutin palmitate & $\mathrm{C}_{16} \mathrm{H}_{32} \mathrm{O}_{2}$ & 847 \\
Rutin stearate & $\mathrm{C}_{18} \mathrm{H}_{36} \mathrm{O}_{2}$ & 875 \\
Rutin oleate & $\mathrm{C}_{18} \mathrm{H}_{34} \mathrm{O}_{2}$ & 873 \\
Rutin linolate & $\mathrm{C}_{18} \mathrm{H}_{32} \mathrm{O}_{2}$ & 871 \\
\hline
\end{tabular}

$\mathrm{R}_{1} / \mathrm{R}_{2} / \mathrm{R}_{3}$ : fatty acid chain.

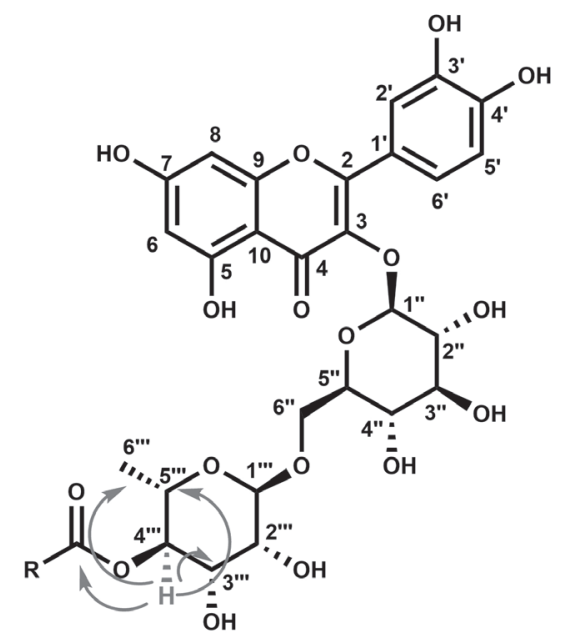

Figure 3. HMBC correlations of H-4"' in rutin ester molecule. 
Some authors ${ }^{9,41}$ have evaluated the selectivity reaction of CALB with rutin, showing that the aglycon of rutin was stabilized at the entrance of the enzyme cavity, while the glycoside was located more internally near to the catalytic residues. The secondary 4"'-OH of the rutin rhamnose was the position that were expected to be acetylated, since it was showed the closest proximity and intrinsic chemical reactivity with the catalytic residues. ${ }^{9,41}$ These data are in agreement with those described in our study, however it does not explain the other esterification positions reported by other authors. ${ }^{18-21}$

\section{Conclusions}

In this study it was prepared rutin derivatives by enzymatic esterification reaction with different saturated and unsaturated fatty acids, achieving good conversion rates. Additionally, the esters were separated for the first time from the reaction mixture via countercurrent chromatography using the biphasic solvent systems consisted by HEMWat (hexane/ethyl acetate/methanol/water), in different proportions. This technique proved to be more advantageous than the traditionally one since it allowed quick isolation and high purity $(\geq 90 \%)$ of the products. It also permitted a substrate recovery for reuse in other enzymatic reactions. Furthermore, the results add valuable information, specially concerning the structures elucidation, not previously described, of different rutin esters.

\section{Supplementary Information}

Supplementary information (thin layer chromatography of partition test, chromatograms, ${ }^{1} \mathrm{H},{ }^{13} \mathrm{C}$, MS of rutin derivatives) is available free of charge at http://jbcs.sbq.org.br as PDF file.

\section{Acknowledgments}

This work was supported by CAPES (PROCADCoordenação de Aperfeiçoamento de Pessoal de Nível Superior-grant No. 179391 and 88881.068489/2014-01), funding from $\mathrm{CNPq}$ (Conselho Nacional de Desenvolvimento Científico e Tecnológico (BR)-grant No. 430752/2016-4) and FAPERJ (Fundação Carlos Chagas Filho de Amparo à Pesquisa do Estado do Rio de Janeiro)-grant No. E-26/202.728/2018.

\section{Author Contributions}

Jéssyca F. O. Couto was responsible for investigation, methodology, visualization, writing, formal analysis, data curation; Daniel Luiz R. Simas for methodology; Marcos Vinicius T. Silva for formal analysis; Thiago Barth for methodology; Shaft C. Pinto for methodology, writingreview and editing; Luzineide W. Tinoco for resources, methodology; Denise Maria G. Freire for resources, conceptualization; Michelle F. Muzitano for supervision, visualization, writing-review and editing, funding acquisition, conceptualization, project administration; Ivana C. R. Leal for supervision, visualization, writingreview and editing, funding acquisition, conceptualization, project administration.

\section{References}

1. Gullón, B.; Lú-Chau, T. A.; Moreira, M. T.; Lema, J. M.; Eibes, G.; Trends Food Sci. Technol. 2017, 67, 220.

2. Panche, A. N.; Diwan, A. D.; Chandra, S. R.; J. Nutr. Sci. 2016, $5,47$.

3. Chua, L. S.; J. Ethnopharmacol. 2013, 150, 805.

4. de Araújo, M. E. M. B.; Franco, Y. E. M.; Messias, M. C. F.; Longato, G. B.; Pamphile, J. A.; Carvalho, P. D. O.; Planta Med. 2017, 83, 7.

5. Viskupicova, J.; Maliar, T.; J. Food Nutr. Res. 2017, 56, 232.

6. Bezborodov, A. M.; Zagustina, N. A.; Appl. Biochem. Microbiol. 2014, 50, 313.

7. Borrelli, G.; Trono, D.; Int. J. Mol. Sci. 2015, 16, 20774.

8. Viskupičová, J.; Šturdík, E.; Ondrejovič, M.; Acta Chim. Slov. 2009, $2,88$.

9. de Oliveira, E. B.; Humeau, C.; Chebil, L.; Maia, E. R.; Dehez, F.; Maigret, B.; Ghoul, M.; Engasser, J. M.; J. Mol. Catal. B: Enzym. 2009, 59, 96.

10. Ardhaoui, M.; Falcimaigne, A.; Ognier, S.; Engasser, J. M.; Moussou, P.; Pauly, G.; Ghoul, M.; J. Biotechnol. 2004, 110, 265.

11. Ardhaoui, M.; Falcimaigne, A.; Engasser, J. M.; Moussou, P.; Pauly, G.; Ghoul, M.; J. Mol. Catal. B: Enzym. 2004, 29, 63.

12. Katsoura, M. H.; Polydera, A. C.; Tsironis, L.; Tselepis, A. D.; Stamatis, H.; J. Biotechnol. 2006, 123, 491.

13. Razak, N. N. A.; Annuar, M. S. M.; Ind. Eng. Chem. Res. 2015 , $54,5604$.

14. Viskupicova, J.; Danihelova, M.; Ondrejovic, M.; Liptaj, T.; Sturdik, E.; Food Chem. 2010, 123, 45.

15. Mellou, F.; Loutrari, H.; Stamatis, H.; Roussos, C.; Kolisis, F. N.; Process Biochem. 2006, 41, 2029.

16. Xanthakis, E.; Theodosiou, E.; Magkouta, S.; Stamatis, H.; Loutrari, H.; Roussos, C.; Kolisis, F.; Pure Appl. Chem. 2010, $82,1$.

17. Duan, Y.; Zongliang, D.; Yao, Y.; Ruixia, L.; Dacheng, W.; J. Agric. Food Chem. 2006, 54, 6219.

18. Nakajima, N.; Ishihara, K.; Itoh, T.; Furuya, T.; Hamada, H.; J. Biosci. Bioeng. 1999, 87, 105. 
19. Ishihara, K.; Nishimura, Y.; Kubo, T.; Okada, C.; Hamada, H.; Nakajima, N.; Plant Biotechnol. 2002, 19, 211.

20. Danieli, B.; Luisetti, M.; Sampognaro, G.; Riva, S.; J. Mol. Catal. B: Enzym. 1997, 177, 193.

21. Mecenas, A. S.; Malafaia, C. R. A.; Sangenito, L. S.; Simas, D. L. R.; Machado, T. B.; Amaral, A. C. F.; Santos, A. L. S.; Freire, D. M. G.; Leal, I. C. R.; PLoS One 2018, 13, e0203159.

22. Kontogianni, A.; Skouridou, V.; Sereti, V.; Stamatis, H.; Kolisis, F. N.; Eur. J. Lipid Sci. Technol. 2001, 103, 655.

23. Gatto, M. T.; Falcocchio, S.; Grippa, E.; Mazzanti, G.; Battinelli, L.; Nicolosi, G.; Lambusta, D.; Saso, L.; Bioorg. Med. Chem. 2002, 10, 269.

24. Almeida, V. M.; Branco, C. R.; Assis, S.; Vieira, I. J.; Braz-Filho, R.; Branco, A.; Chem. Cent. J. 2012, 6, 41.

25. Ziaullah; Bhullar, K. S.; Warnakulasuriya, S. N.; Rupasinghe, H. P.; Bioorg. Med. Chem. 2013, 21, 684.

26. Mellou, F.; Lazari, D.; Skaltsa, H.; Tselepis, A. D.; Kolisis, F. N.; Stamatis, H.; J. Biotechnol. 2005, 116, 295.

27. Salem, J. H.; Humeau, C.; Chevalot, I.; Harscoat-Schiavo, C.; Vanderesse, R.; Blanchard, F.; Fick, M.; Process Biochem. 2010, 45,382 .

28. Chebil, L.; Anthoni, J.; Humeau, C.; Gerardin, C.; Engasser, J. M.; Ghoul, M.; J. Agric. Food Chem. 2007, 55, 9496.

29. Lue, B. M.; Guo, Z.; Glasius, M.; Xu, X.; J. Am. Oil Chem. Soc. 2010, 87, 55.
30. Zheng, M. M.; Wang, L.; Huang, F. H.; Guo, P. M.; Wei, F.; Deng, Q. C.; Zheng, C.; Wan, C. Y.; J. Mol. Catal. B: Enzym. 2013, 95, 82.

31. Kontogianni, A.; Skouridou, V.; Sereti, V.; Stamatis, H.; Kolisis, F. N.; J. Mol. Catal. B: Enzym. 2003, 21, 59.

32. Conway, W. D.; J. Chromatogr. 1991, 538, 27.

33. Costa, F. D. N.; Leitão, G. G.; J. Sep. Sci. 2010, 33, 336.

34. Friesen, J. B.; Pauli, G. F.; J. Chromatogr. A 2007, 1151, 51.

35. Chebil, L.; Humeau, C.; Anthony, J.; Dehez, F.; Engasser, J. M.; Ghoul, M.; J. Chem. Eng. Data 2007, 52, 1552.

36. Passicos, E.; Santarelli, X.; Coulon, D.; Biotechnol. Lett. 2004, 26, 1073.

37. Cui, H. Y.; Jia, X. Y.; Zhang, X.; Zhang, J.; Zhang, Z. Q.; Sep. Purif. Technol. 2011, 77, 269.

38. Ito, Y.; J. Chromatogr. A 2005, 1065, 145.

39. Wuillda, A. C. J. D. S.; Trovó, M.; Martins, C. C.; Costa, N.; Rev. Virtual Quim. 2018, 10, 672.

40. Xu, Y.; Lv, X.; Yang, G.; Zhan, J.; Li, M.; Long, T.; Ho, C.; Food Chem. 2019, 292, 160.

41. de Oliveira, E. B.; Humeau, C.; Maia, E. R.; Chebil, L.; Ronat, E.; Monard, G.; Ruiz-Lopez, M. F.; Ghoul, M.; Engasser, J.; J. Mol. Catal. B: Enzym. 2010, 66, 325.

Submitted: April 6, 2020

Published online: October 14, 2020 